\title{
MULTI-USER OFDM EMPLOYING PARALLEL INTERFERENCE CANCELLATION ASSISTED DECISION-DIRECTED CHANNEL ESTIMATION
}

\author{
Matthias Münster and Lajos Hanzo \\ Dept. of ECS, University of Southampton, SO17 1BJ, UK. \\ Tel: +44-23-80-593 125, Fax: +44-23-80-594 508 \\ Email:1h@ecs.soton.ac.uk, http://www-mobile.ecs.soton.ac.uk
}

\begin{abstract}
Multiple reception antenna assisted Space Division Multiple Access (SDMA) techniques designed for wireless Orthogonal Frequency Division Multiplexing (OFDM) systems have recently drawn wide interests. These techniques facilitate the implementation of effective multi-user detection algorithms at the receiver. A prerequisite of their operation is the availability of an estimate of the channels' frequency domain transfer functions. This contribution analyses a recursive Decision-Directed Channel Estimator (DDCE). Our simulation results demonstrate that this channel transfer function estimator is capable of outperforming state-of-the-art transversal filter based DDCEs in terms of the mean-square estimation error in scenarios, where the product of the number of transmit antennas and the number of CIR taps to be estimated per transmit antenna approaches the number of subcarriers hosted by an OFDM symbol.
\end{abstract}

\section{OVERVIEW}

In this contribution an improved decision-directed channel estimator (DDCE) designed for multi-user Space Division Multiple Access (SDMA) assisted Orthogonal Frequency Division Multiplexing (OFDM) applications is presented. The structure of the paper is as follows. In Section 2 the SDMA scenario is outlined, followed by the portrayal of the channel estimation problem and the review of state-of-the-art solutions in Section 3.1. In Section 3.2 the proposed recursive DDCE is analysed with the aim of deriving an expression for the á priori channel transfer function estimation Mean-Square Error (MSE) at the input of the antenna combining stage. A further expression is provided for the á posteriori MSE encountered after updating the channel transfer function estimates on the basis of the most recently received subcarrier symbols. Furthermore, the recursive estimator's stability conditions are derived and potential approaches for determining the optimum á priori channel estimator's coefficients in the sense of the Minimum MSE (MMSE) are presented. This is followed by our performance assessment in Section 4. Our conclusions will be offered in Section 5 .

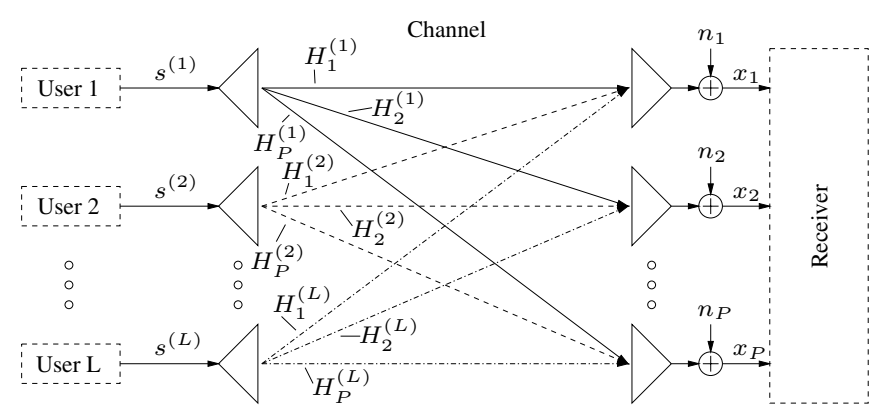

Figure 1: Schematic of an SDMA uplink scenario as observed on an OFDM subcarrier basis, where each of the $L$ users is equipped with a single transmit antenna and the receiver is assisted by a $P$-element antenna front-end.

\section{THE SIGNAL MODEL}

In Figure 1 we have portrayed an SDMA uplink transmission scenario, where each of the $P$ simultaneous users is equipped with a single transmission antenna, while the receiver capitalizes on a $P$ element antenna front-end. The set of complex signals, $x_{p}[n, k]$, $p \in\{1, \ldots, P\}$ received by the $P$-element antenna array in the $k$-th subcarrier of the $n$-th OFDM symbol is constituted by the superposition of the independently faded signals associated with the $L$ users sharing the same space-frequency resource. The received signal was corrupted by the Gaussian noise at the array elements. Regarding the statistical properties of the different signal components depicted in Figure 1, we assume that the complex data signal $s^{(l)}$ transmitted by the $l$-th user has zero-mean and a variance of $\sigma_{l}^{2}$. The AWGN noise process $n_{p}$ at any antenna array element $p$ exhibits also zero-mean and a variance of $\sigma^{2}$, which is identical for all array elements. The frequency domain channel transfer functions $H_{p}^{(l)}$ of the different array elements $p \in\{1, \ldots, P\}$ or users $l \in\{1, \ldots, L\}$ are independent, stationary, complex Gaussian distributed processes with zero-mean and unit variance.

VTC'2002, VANCOUVER, CANADA

The financial support of the European Union and that of the EPSRC, Swindon, UK is gratefully acknowledged 


\section{PARALLEL INTERFERENCE CANCELLATION ASSISTED DECISION-DIRECTED CHANNEL ESTIMATOR}

\subsection{Motivation}

A Minimum Mean-Square Error (MMSE) channel estimator was proposed by Li et al. [1], which aims at recovering the different users' channel transfer functions on the basis of the output signal of a specific reception antenna element and by also capitalizing on the remodulated received symbols associated with the different users. The performance of this estimator was found to be limited in terms of the mean-square estimation error in scenarios, where the product of the number of transmit antennas and the number of CIR taps to be estimated per transmit antenna approaches the number of subcarriers hosted by an OFDM symbol.

In [2] a DDCE was proposed by Jeon et al. for a space-time coded OFDM scenario of two transmit antennas and two receive antennas. Specifically, the channel transfer function associated with each transmit-receive antenna pair was estimated on the basis of the output signal of the specific receive antenna upon subtracting the interfering signal contributions associated with the remaining transmit antennas. These interference contributions were estimated by capitalizing on the knowledge of the channel transfer functions of all interfering transmit antennas predicted during the $(n-1)$-th OFDM symbol period for the $n$-th OFDM symbol, also invoking the corresponding remodulated symbols associated with the $n$-th OFDM symbol.

By contrast, in [3] a similar technique was proposed by Li with the aim of simplifying the DDCE approach of [1], which operates in the time-domain. Since according to our analysis the subtractive DDCE approach proposed in [3], where the cancellation of the different transmit antennas' interfering signals is performed in the time-domain, exhibits a higher complexity than the subtractive DDCE of [2] performing the interference cancellation in the frequency domain, we decided to employ the latter approach in our further investigations.

\subsection{The Recursive Estimator}

\subsection{1. Á Priori and Á Posteriori Channel Estimates}

The complex output signal $x_{p}[n, k]$ of the $p$-th receiver antenna element in the $k$-th subcarrier of the $n$-th OFDM symbol is given by:

$$
x_{p}[n, k]=\sum_{i=1}^{L} H_{p}^{(i)}[n, k] s^{(i)}[n, k]+n_{p}[n, k],
$$

where the different variables have been defined in Section 2. An $a$ posteriori (apt) estimate $\hat{H}_{a p t}^{(j)}[n, k]$ of the channel transfer factor $H^{(j)}[n, k]$ between the $j$-th user's single transmit antenna and the $p$-th receive antenna can be obtained by subtracting all the $(L-1)$ interfering users' estimated signal contributions from the composite received signal $x_{p}[n, k]$ of the $L$ users. This is followed by normalization of the resultant expression to the $j$-th user's detected complex symbol $\hat{s}^{(j)}[n, k]$, yielding:

$$
\hat{H}_{a p t}^{(j)}[n, k]=\frac{1}{\hat{s}^{(j)}[n, k]}\left(x[n, k]-\sum_{\substack{i=1 \\ i \neq j}}^{L} \hat{H}_{a p r}^{(i)}[n, k] \hat{s}^{(i)}[n, k]\right),
$$

where for simplicity's sake we have omitted the receiver antenna's index $p$. In Equation 2, $\hat{H}_{a p r}^{(i)}[n, k]$ denotes the complex á priori channel transfer factor predicted during the $(n-1)$-th OFDM symbol period for the $k$-th subcarrier of the $n$-th OFDM symbol, as a function of the á posteriori channel transfer factors $\hat{H}_{a p t}^{(i)}[n-\dot{n}, \hat{k}]$ associated with the previous $N$ number of OFDM symbols, which is formulated as:

$$
\hat{H}_{a p r}^{(\hat{i})}[n, k]=f\left(\hat{H}_{a p t}^{(\hat{i})}[n-1, \hat{k}], \ldots, \hat{H}_{a p t}^{(\hat{i})}[n-N, \hat{k}]\right),
$$

where $i \in\{1, \ldots, L\}, \dot{k} \in\{0, \ldots, K-1\}$. We will further elaborate on the structure of the function $f()$ in Section 3.2.4. Upon assuming error-free symbol decisions we have $\hat{s}^{(l)}[n, k]=$ $s^{(l)}[n, k], l \in\{1, \ldots, L\}$ and substituting $x[n, k]$ defined in Equation 1 into Equation 2 yields:

$$
\begin{aligned}
\hat{H}_{a p t}^{(j)}[n, k]= & H^{(j)}[n, k]+\frac{n[n, k]}{s^{(j)}[n, k]}+ \\
& +\sum_{\substack{i=1 \\
i \neq j}}^{L}\left(H^{(i)}[n, k]-\hat{H}_{a p r}^{(i)}[n, k]\right) \frac{s^{(i)}[n, k]}{s^{(j)}[n, k]} .
\end{aligned}
$$

From Equation 4 we infer that the á posteriori channel estimate $\hat{H}_{a p t}^{(j)}[n, k]$ deviates from its true value $H^{(j)}[n, k]$ due to contamination by the AWGN as well as by an additional contribution attributed to the imperfections of the á priori estimates $\hat{H}_{a p r}^{(i)}[n, k]$ of the channel transfer factors $H^{(i)}[n, k], i \neq j$.

\subsection{2. Á Priori and Á Posteriori Channel Estimation MSE without Filtering}

Following from Equation 4 a relation between the $j$-th á posteriori channel estimation error signal $\Delta \hat{H}_{a p t}^{(j)}[n, k]$ and the $(L-1) a^{\prime}$ priori channel estimation error signals $\Delta \hat{H}_{a p r}^{(i)}[n, k]$, with $i \neq j$ is hence given by:

$$
\begin{aligned}
\Delta \hat{H}_{a p t}^{(j)}[n, k]= & -\frac{n[n, k]}{s^{(j)}[n, k]}- \\
& -\sum_{\substack{i=1 \\
i \neq j}}^{L}\left(\Delta \hat{H}_{a p r}^{(i)}[n, k]\right) \frac{s^{(i)}[n, k]}{s^{(j)}[n, k]},
\end{aligned}
$$

where

$$
\Delta \hat{H}_{a p(t / r)}^{(\hat{i})}[n, k]=H^{(\hat{i})}[n, k]-\hat{H}_{a p(t / r)}^{(\hat{i})}[n, k],
$$

$i \in\{1, \ldots, L\}$. The variance of the error signals $\Delta \hat{H}_{a p t}^{(j)}[n, k]$ and $\Delta \hat{H}_{a p r}^{(i)}[n, k], i \neq j$ or, equivalently, the mean-square channel estimation errors are hence related by:

$$
M S E_{a p t}^{(j)}[n, k]=\alpha \cdot \frac{\sigma^{2}}{\sigma_{j}^{2}}+\alpha \cdot \sum_{\substack{i=1 \\ i \neq j}}^{L} M S E_{a p r}^{(i)}[n, k],
$$

where

$$
M S E_{a p(t / r)}^{(\hat{i})}[n, k]=E\left\{\left|\Delta \hat{H}_{a p(t / r)}^{(\hat{i})}[n, k]\right|^{2}\right\},
$$

$i \in\{1, \ldots, L\}$. In Equation $7 \alpha$ denotes the so-called "modulation noise enhancement factor" [4], defined as $\alpha=E\left\{\left|1 / s^{()}[n, k]\right|^{2}\right\}$. For M-ary Phase Shift Keying (MPSK) modulation schemes, such 
as for example QPSK we have $\alpha=1$, while for higher-order Quadrature Amplitude Modulation (QAM) schemes we have $\alpha>$ 1 [4]. In the context of the derivation of Equation 7 we have exploited that the complex symbols transmitted by different users in the same subcarrier are statistically independent, which also implies that they are uncorrelated, i.e. $E\left\{s^{(i)}[n, k] s^{(j)^{*}}[n, k]\right\}=0$, $i \neq j$.

\subsection{3. Á Priori and Á Posteriori Channel Estimation MSE after FFT based CIR Windowing}

In order to further improve the channel transfer function estimator's MSE, a well-established approach [4,5] is to apply Fast Fourier Transform (FFT) based time-domain windowing to the á posteriori estimated channel transfer factors $\hat{H}_{a p t}^{(j)}[n, k], k \in\{0, \ldots, K-$ $1\}$ of Equation 4, using a window size $K_{0}$ equivalent to $\left\lceil T_{m} / T_{s}\right\rceil$, where $T_{m}$ denotes the multipath spread of the channel and $T_{s}$ is the samping period duration. Hence in the simplified scenario of a sample-spaced CIR the desired signal component $H^{(j)}[n, k]$ of Equation 4 is not affected by the windowing, while the variance of the undesired á posteriori channel estimation error signal $\Delta \hat{H}_{a p t}^{(j)}[n, k]$ of Equation 5 - which was quantified in Equation 7 is reduced by a factor of $\rho=\frac{K_{0}}{K}$. The factor $\rho$ was termed in [4] as the "filter noise reduction factor". The MSE of Equation 7 can therefore be modified to yield:

$$
M \dot{S} E_{\text {apt }}^{(j)}[n, k]=\chi \cdot \frac{\sigma^{2}}{\sigma_{s}^{2}}+\chi \cdot \sum_{\substack{i=1 \\ i \neq j}}^{L} M \dot{S} E_{a p r}^{(i)}[n, k],
$$

where $\chi=\rho \alpha$. In contrast to Equation 7, the reduced MSE is denoted by $M \dot{S} E$. Please also note that in all previous equations the variables of estimated channel transfer functions $\hat{H}$ have to be substituted by their time-domain windowed counterparts. It is of critical importance that due to the windowing not only the variance of the spectrally white AWGN contribution $n[n, k]$, but also the variance of the spectrally coloured a priori channel estimation error $\Delta \hat{H}_{a p r}^{(i)}[n, k]$ of Equation 5 is reduced by a factor of $\rho$ during the $n$-th OFDM symbol period. The latter reduction is observed, since although the CIR resulting in the frequency domain channel transfer function $\hat{H}_{a p r}^{(i)}[n, k]$ has been truncated to the lowest-delay $K_{0}$ number of CIR taps as a result of the windowing during the $(n-1)$-th OFDM symbol period, nonetheless the multiplication of $\Delta \hat{H}_{a p r}^{(i)}[n, k]$ with the complex subcarrier symbols $s^{(i)}[n, k]$ in Equation 5 - which are assumed to be statistically independent for different subcarriers and different OFDM symbols - results in the spreading of its effects over all CIR taps in the time-domain. Thus the á priori channel transfer function estimation error $\Delta \hat{H}_{a p r}^{(i)}[n, k]$ can be further reduced by the time-domain windowing invoked during the $n$-th OFDM symbol period.

\subsection{4. Á Priori and Á Posteriori Channel Estimation MSE af- ter FFT based CIR Windowing in the Context of N-Tap Linear Á Priori Channel Estimation}

As observed in Equations 7 and 9, the á posteriori channel transfer function estimation MSE in the $n$-th OFDM symbol is linked to the á posteriori channel estimation MSE in the $(n-1)$-th OFDM symbol via the á priori channel estimation or prediction formula of Equation 3.
Hence, instead of Equation 3 for an $N$-tap linear á priori channel transfer factor estimator we obtain:

$$
\dot{\hat{H}}_{a p r}^{(\hat{i})}[n, k]=\sum_{\dot{n}=1}^{N} \xi_{\dot{n}}^{(\dot{i})} \dot{\hat{H}}_{a p t}^{(\hat{i})}[n-\dot{n}, k],
$$

where $i \in\{1, \ldots, L\}$. Since different users may exhibit different maximum Doppler frequencies due to their different vehicular speeds, each user is associated with an individual set of CIR- or channel transfer factor predictor coefficients. More specifically, as observed in Equation 10, the $i$-th user's predictor coefficients are given by $\xi_{n}^{\left(\hat{n}^{\prime}\right)}, \dot{n} \in\{1, \ldots, N\}$. Upon substituting Equation 10 into Equation 6 and further substituting the result into Equation 8 we obtain:

$$
\begin{aligned}
M \dot{S} E_{a p r}^{(\hat{i})}[n, k]= & M \dot{S} E_{\text {Decorr }}^{(\hat{i})}[n, k]+ \\
& +\sum_{n=1}^{N}\left(\xi_{\dot{n}}^{(\hat{i})}\right)^{2} M \dot{S} E_{a p t}^{(\hat{i})}[n-\dot{n}, k],
\end{aligned}
$$

where

$$
\begin{aligned}
& M \dot{S} E_{\text {Decorr }}^{(\dot{i})}[n, k]=E\left\{\mid\left(H^{(\hat{i})}[n, k]-\right.\right. \\
& \left.-\left.\sum_{n=1}^{N} \xi_{\dot{n}}^{(\dot{i})} H^{(i)}[n-\dot{n}, k]\right|^{2}\right\}
\end{aligned}
$$

denotes the channel transfer function mismatch MSE due to the channel's decorrelation. In order to separate the total MSE into its components $M \dot{S} E_{\text {Decorr }}^{(\hat{i})}[n, k]$ and $M \dot{S} E_{\text {apt }}^{(\hat{i})}[n-\dot{n}, k], \dot{n} \in$ $\{1, \ldots, N\}$, as seen in Equation 11, we exploited that the expected value of the transmitted signal is zero. Specifically, we have $E\left\{s^{()}[]\right\}=0$, which is a consequence of the symmetric nature of the modulation constellations considered, as well as that of the statistically independent nature of the complex symbols transmitted in the same subcarrier of different OFDM symbols, which is expressed as $E\left\{s^{(\hat{i})}\left[\dot{n}_{1}, k\right] \cdot s^{(\hat{i})^{*}}\left[\dot{n}_{2}, k\right]\right\}=0$ and $\dot{n}_{1}, \dot{n}_{2} \in$ $\{1, \ldots, N\}, \dot{n}_{1} \neq \dot{n}_{2}$.

\subsubsection{Stability Analysis}

On the basis of Equations 9, 11 and 12 a closed-form solution can be obtained for the á priori and á posteriori MSE associated with the different users' channel transfer function estimation processes as a function of both the first-order statistics of the different users' channel transfer functions, as indicated by Equation 12, as well as a function of the prediction filter coefficients employed and that of the parameter $\chi=\rho \alpha$, where $\rho=\frac{K_{0}}{K}$. These MSE expressions are derived by stipulating that for each user the system is in its steady-state condition expressed as:

$$
M \dot{S} E_{a p(t / r)}^{(\hat{i})}[n, k]=M \dot{S} E_{a p(t / r)}^{(\hat{i})}[n-\dot{n}, k],
$$

where $i \in\{1, \ldots, L\}$ and $\dot{n} \in\{1, \ldots, N\}$. In order to simplify the analysis we will assume that all the users have an identical transmit power of $\sigma_{i}^{2}=\sigma_{s}^{2}, i \in\{1, \ldots, L\}$, and that the different users' channels exhibit the same maximum Doppler frequency. Hence their prediction filter coefficients and consequently also their channel transfer function estimation MSE in the above steady-state condition will be identical, yielding:

$$
M \ddot{M} E_{a p(t / r)}^{(\grave{i})}[n-\grave{n}, k]=M \ddot{S} E_{a p(t / r)}^{(\grave{j})}[n-\grave{n}, k],
$$


where $i, \dot{j} \in\{1, \ldots, L\}$ and $\grave{n} \in\{0, \ldots, N\}$. With the aid of these assumptions, we obtain for the specific á posteriori channel estimation MSE:

$$
M \ddot{S} E_{a p t}[n, k]=\frac{\chi \cdot \frac{\sigma^{2}}{\sigma_{s}^{2}}+\chi \cdot(L-1) \cdot M \ddot{S} E_{D e c o r r}[n, k]}{1-\chi \cdot(L-1) \cdot \xi}
$$

and for the specific á priori channel estimation MSE:

$$
M \ddot{S} E_{a p r}[n, k]=\frac{\chi \cdot \xi \cdot \frac{\sigma^{2}}{\sigma_{s}^{2}}+M \ddot{S} E_{D e c o r r}[n, k]}{1-\chi \cdot(L-1) \cdot \xi},
$$

where $\xi=\sum_{\dot{n}=1}^{N} \xi_{\dot{n}}^{2}$. The steady-state stability condition can be directly inferred from the denominator of Equations 15 and 16 by exploiting that the channel transfer function estimation MSE must be a finite positive value according to Equation 8, yielding:

$$
\chi \cdot(L-1) \cdot \xi<1 .
$$

This is equivalent to the condition that the poles of the system's transfer function are located inside the z-transform related unit circle.

\subsubsection{MMSE Optimum Á Priori Estimator Coefficients}

A viable approach to determining the á priori channel estimator's coefficients $\xi_{n}, \dot{n} \in\{1, \ldots, N\}$ seen in Equation 10 is to minimize the MSE of the á priori channel transfer function estimates, as observed at the input of the detection stage. More specifically, for the simplified scenario of Section 3.2.5, this involves minimizing Equation 16, for example with the aid of standard gradient based techniques. For á priori channel estimator orders higher than one in Equation 16 it is not straightforward to find a closedform solution for the optimum estimator coefficients. Hence a more practical solution is to optimize these coefficients with the aid of an iterative approach, such as for example the gradient based method [6], or a genetic algorithm [7] as invoked in the context of our numerical evaluations in Section 4. For an insufficient number of filter taps the cost function given by Equation 16 exhibits multiple local minima associated with potentially different values [8] and hence the gradient approach may converge to a suboptimum set of estimator coefficients. By contrast, genetic algorithms are less likely to get trapped at a suboptimum solution due to their ability to more thoroughly explore the solution space.

\section{SIMULATION RESULTS}

The OFDM parameters stipulated were $K=512$ subcarriers and a guard interval length of $K_{0}=64$ samples - following the specifications of an indoor WATM system [6]. The OFDM symbol normalized Doppler frequency was assumed to be $F_{D}=0.007$, which corresponds to a vehicular speed of $50 \mathrm{~km} / \mathrm{h}$ in the context of the indoor WATM system's parameters. Throughout our investigations we will capitalize on the assumption frame-invariant fading in order to avoid the obfuscating effects of inter-subcarrier interference (ICI) and also on the assumption of error-free subcarrier symbol decisions, which is justified for sufficiently high SNRs in the context of effective SDMA multiuser detection algorithms, such as for example successive interference cancellation (SIC) $[9,10]$.

In Figure 2 we have plotted the á priori estimator MSE of Equation 16 exhibited in the context of the optimum filter weights
Fr.-Inv. Fad. SWATM, L Rec.-Antennas, L Users, MPSK

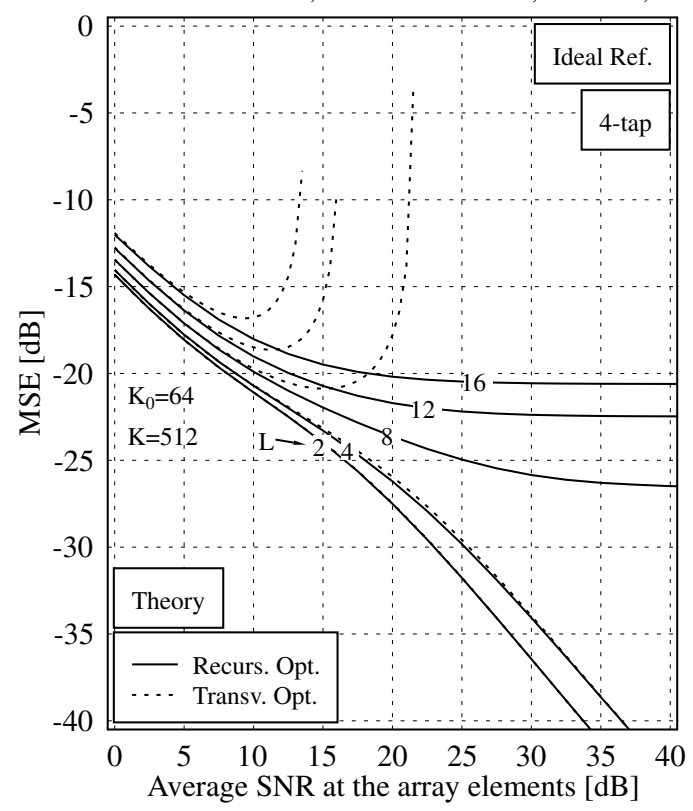

Figure 2: Á priori channel estimation MSE $\left(\sigma^{2}=2\right)$ of the recursive $N=4$-tap á priori estimation assisted arrangement, assuming error-free subcarrier symbol decisions, parametrized with the number of simultaneous users $L$. A further MSE improvement can be achieved upon increasing the á priori channel estimator's range.

taking into account the recursive nature of the system, as well as that exhibited in the context of the suboptimum transversal filter weights, derived from Equation 16 by removing the signal feedback related term $\chi \cdot(L-1) \cdot \xi$, both as a function of the number of simultaneous users $L$. An á priori channel transfer function estimator range of four taps was assumed. We observe that for the suboptimum filter weights the estimator suffers from an instability, while for the optimum recursive filter weights stability problems do not occur. Along with increasing the number $L$ of simultaneous users the estimator MSE is degraded, since more noise is injected into the estimation process due to the channel transfer function decorrelation between successive OFDM symbols.

In order to further underline the potential benefits of the advocated recursive estimator structure in scenarios supporting a relatively high number of users and transmit antennas, in Figure 3 we have compared the á priori MSE exhibited by the recursive estimator to the $a ́$ priori MSE exhibited by a transversal channel transfer function estimator, which followed the philosophy of [1]. We note that here a scenario of $K=64$ and $K_{0}=8$ is considered which is identical to a scenario of $K=512$ and $K_{0}=64$ in the context of the recursive estimator advocated, in terms of the á priori estimator MSE, provided that a sample-spaced CIR is used. This is, because the a priori MSE is a function of the "filter noise reduction factor" $\rho=\frac{K_{0}}{K}$, which is incorporated in the variable $\chi$, as observed in Equation 16. In Figure 3 we observe that for both estimators the á priori MSE is degraded upon increasing the number $L$ of simultaneous SDMA users, each supported by a single transmit antenna. Particularly, for a scenario of $L=8$ simultaneous users the MSE exhibited by the transversal estimator of [1] is severely degraded, which is due to the limited invertibility of the 
Fr.-Inv. Fad., L Rec.-Antennas, L Users, MPSK

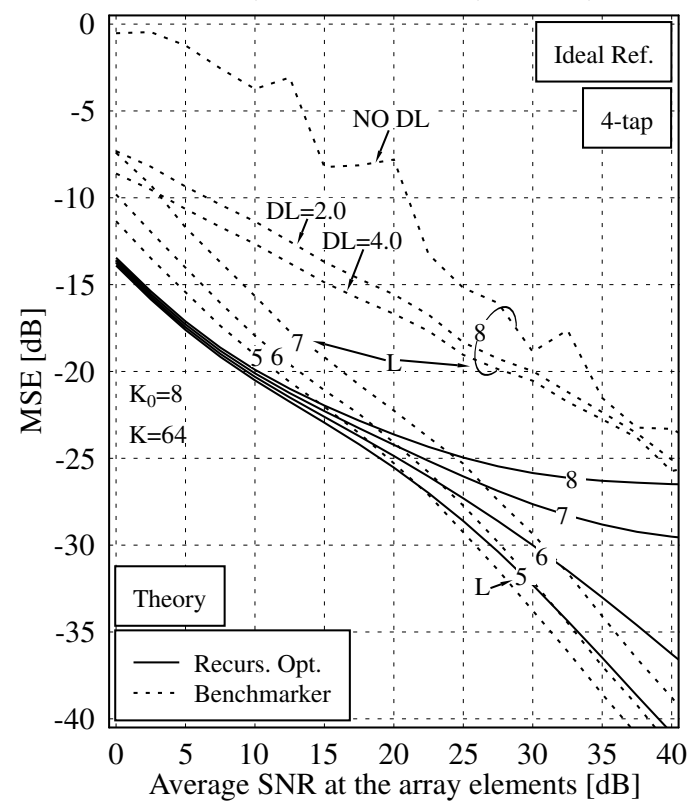

Figure 3: Á priori channel estimation MSE $\left(\sigma^{2}=2\right)$ of the recursive $N=4$-tap á priori estimation assisted arrangement, assuming error-free symbol decisions, parametrized with the number of simultaneous users $L$. As a benchmarker we have plotted the á priori channel estimation MSE $\left(\sigma^{2}=2\right)$ of a transversal $N=4$ tap á priori estimation assisted arrangement [1], again, upon assuming error-free subcarrier symbol decisions, where in the context of $L=8$ simultaneous users a simple matrix regularization by diagonal loading (DL) was employed for facilitating the invertibility of the different users' transmitted symbol sequences' correlation matrix.

correlation matrix associated with the different users' transmitted symbol sequences. By even further increasing the number of simultaneous users, namely beyond the value of $L_{\max }=K / K_{0}$, reliable channel estimation became virtually impossible. This was also alluded to in [3]. By contrast, the recursive estimator proposed here does not suffer from these limitations.

\section{CONCLUSIONS}

In this contribution we have analysed and assessed a subtractive recursive DDCE proposed for multiuser OFDM-SDMA environments. As a performance criterion the á priori estimator MSE was employed. We have demonstrated that the recursive estimator is capable of operating in scenarios where the product of the $L$ number of transmit antennas supported and the number of CIR taps $K_{0}$ to be estimated per channel is in excess of the number of subcarriers $K$, i.e. $L>K / K_{0}$. This was found to be a limitation for the transversal channel transfer function estimator of [1]. Furthermore, the complexity of the recursive estimator which performs the interference cancellation in the frequency domain is significantly lower than that of the transversal estimator of [1] and it is also lower than that of a subtractive recursive estimator operating in the time-domain, which was proposed in [3]. A disadvantage associated with the recursive approach is related to the more complex channel transfer function filter coefficient calculation, which was performed in the context of our numerical simulations with the aid of a genetic algorithm.

\section{REFERENCES}

[1] Y. Li, N. Seshadri, and S. Ariyavisitakul, "Channel Estimation for OFDM Systems with Transmitter Diversity in Mobile Wireless Channels," IEEE Journal on Selected Areas in Communications, vol. 17, pp. 461-471, March 1999.

[2] W. G. Jeon, K. H. Paik, and Y. S. Cho, "An Efficient Channel Estimation Technique for OFDM Systems with Transmitter Diversity," in Proc. of PIMRC IEEE, 2000.

[3] Y. Li, "Optimum Training Sequences for OFDM Systems with Multiple Transmit Antennas," in Proc. of Global Telecommunications Conference, vol. 3, (San Francisco, United States), pp. 1478-1482, IEEE, November 27 - December 1 2000 .

[4] A. Chini, Y. Wu, M. El-Tanany, and S. Mahmoud, "Filtered Decision Feedback Channel Estimation for OFDM Based DTV Terrestrial Broadcasting System," IEEE Transactions on Broadcasting, vol. 44, pp. 2-11, March 1998.

[5] Y. Li, L. J. Cimini, and N. R. Sollenberger, "Robust Channel Estimation for OFDM Systems with Rapid Dispersive Fading Channels," IEEE Transactions on Communications, vol. 46, pp. 902-915, Apr 1998.

[6] L. Hanzo, W. Webb, and T. Keller, Single- and Multi-carrier Quadrature Amplitude Modulation. IEEE Press- John Wiley, April 2000.

[7] Y. Kai and L. Hanzo, " Genetic Algorithm Assisted Joint Multiuser Symbol Detection and Fading Channel Estimation for Synchronous CDMA Systems," IEEE JSAC, vol. 19, pp. 985-998, June 2001.

[8] O. Macchi, The Least Mean Squares Approach with Applications in Transmission. John Wiley, New York, 1995.

[9] G. D. Golden, G. J. Foschini, R. A. Valenzuela, and P. W. Wolniansky, "Detection Algorithms and Initial Laboratory Results using V-BLAST Space-Time Communication Architecture," IEE Electronics Letters, vol. 35, pp. 14-16, Jan. 1999.

[10] M. Münster and L. Hanzo, "Co-Channel Interference Cancellation Techniques for Antenna Array Assisted Multiuser OFDM Systems," in Proc. of 3G-'2000 Conference, vol. 1, (London, Great Britain), pp. 256-260, IEE, March 27-29 2000 